SISSA-71/2009/EP

ULB-TH/09-40

\title{
Patterns of Soft Masses from General Semi-Direct Gauge Mediation
}

\author{
Riccardo Argurio $^{1}$, Matteo Bertolini ${ }^{2}$, Gabriele Ferretti ${ }^{3}$ \\ and Alberto Mariotti ${ }^{4}$ \\ ${ }^{1}$ Physique Théorique et Mathématique and International Solvay Institutes \\ Université Libre de Bruxelles, C.P. 231, 1050 Bruxelles, Belgium \\ 2 SISSA and INFN - Sezione di Trieste \\ Via Beirut 2; I 34014 Trieste, Italy \\ ${ }^{3}$ Department of Fundamental Physics \\ Chalmers University of Technology, 41296 Göteborg, Sweden \\ 4 Theoretische Natuurkunde and International Solvay Institutes \\ Vrije Universiteit Brussel, Pleinlaan 2, B-1050 Brussels, Belgium
}

\begin{abstract}
We give a general formulation of semi-direct gauge mediation of supersymmetry breaking where the messengers interact with the hidden sector only through a weakly gauged group. Using this general formulation, we provide an explicit proof that the MSSM gaugino masses are vanishing to leading order in the gauge couplings. On the other hand, the MSSM sfermion masses have, generically, a non-vanishing leading contribution. We discuss how such a mechanism can successfully be combined with other mediation schemes which give tachyonic sfermions, such as sequestered anomaly mediation and some direct gauge mediation models.
\end{abstract}




\section{Introduction}

Gauge mediation [1] (see [2] for a comprehensive review) is a popular mechanism to mediate supersymmetry breaking from a hidden sector, where the breaking occurs, to the visible sector (the MSSM or extensions thereof). Its main virtue is, besides calculability, that of naturally implementing a strong suppression of all soft terms leading to flavor changing neutral currents. In this framework, the only degrees of freedom of the MSSM which interact with the hidden sector are those of the gauge sector.

In exploring the phenomenological implications of various mechanisms of supersymmetry breaking, it is desirable to isolate those predictions that are independent of the specific details of the model. Recently, a general formulation of gauge mediation (GGM) was given that accomplishes this in a very explicit way [3]. In this formalism, gauge mediation is formulated in terms of the currents (both fermionic and bosonic) that couple to the gauge degrees of freedom. It can be shown that the spectrum of soft masses is restricted by two sum rules for the sfermions but is otherwise generic. For instance, these sum rules do not specify any pattern between the masses of gauginos and the masses of the sfermions, which is thus an undefined feature in a generic gauge mediation model. For a discussion of such patterns and hierarchies, see e.g. [4, 5].

In practice, specific models of gauge mediation will prominently feature messenger superfields, which are those fields charged under the MSSM gauge groups that also interact with the supersymmetry breaking sector. Once a given model is specified, patterns and hierarchies among the soft terms immediately arise. As an example, the minimal gauge mediation scenario (MGM) [6] (often used in phenomenological applications) has messenger chiral superfields which couple trilinearly with a spurion that provides them with both a supersymmetric mass and an off-diagonal non supersymmetric mass. In this model the gaugino and sfermion masses turn out to be of the same order of magnitude. Other examples, which are denoted direct gauge mediation models (DGM) 7], have messengers which are typically composite fields directly participating in the dynamical supersymmetry breaking mechanism. Clearly, those would be the most appealing models (solving the hierarchy problem 
with no tuned parameters), but it turns out that often in such models the gaugino masses are highly suppressed or the sfermion masses are tachyonic. In addition, supersymmetry breaking generally requires a large hidden gauge group giving rise to a Landau pole in the visible couplings. Though models which cure some of these problems exist (possibly based on metastable hidden sectors, see e.g. [8]), it would be desirable to single out general properties of models which are phenomenologically viable.

We focus on a class of models where the messengers interact with the hidden sector only through (non-MSSM) gauge interactions with gauge group $G_{h}$ and coupling $g_{h}$ but, unlike DGM, they do not participate to the supersymmetry breaking dynamics. These models were dubbed semi-direct gauge mediation (SDGM) in [9]. A subclass of these models - characterized by the further requirement that letting the hidden coupling $g_{h} \rightarrow 0$ did not lead to restoration of supersymmetry in the hidden sector - was considered in [10] under the name of mediator models. Mediator models obey directly all the conditions of GGM [3] if one considers the messengers as part of the visible sector. Our computations are done mainly with this class of models in mind. We believe, however, that our analysis can be extended even to the class of models of SDGM considered in [9] - characterized by the fact that supersymmetry breaking requires $g_{h} \neq 0$ - as long as one is allowed to treat perturbatively the masses of the hidden gauge multiplets, although a full analysis of the issues involved in higgsed gauged mediation is beyond the scope of the present paper.

SDGM models lie somewhat in between minimal and direct gauge mediation. Like MGM they have an explicit messenger sector. Like DGM, however, no spurionlike coupling is needed and everything is mediated by gauge interactions alone. The only superpotential term for the messenger field is a mass term. From a theoretical point of view the interest of such models lies in their simplicity, and also in the rather straightforward way in which they can be generated in string theory inspired quiver gauge theories (in which also the mass term arises dynamically, without the need to be introduced as an external fine tuned parameter) [11]. An important advantage of these models with respect to DGM is that they ameliorate the Landau pole problem which often afflicts DGM models, since $G_{h}$ can be as small as $U(1)$.

Our goal is to discuss the generic features of this class of models by implementing 
a formalism very similar to the one of GGM [3]. Our approach will be general since we parameterize the supersymmetry breaking sector by currents instead of by a spurion. We aim at computing general expressions relating the MSSM gaugino and sfermion masses to the correlators of the supersymmetry breaking currents.

The outcome of our analysis is as follows.

The gaugino masses are vanishing at the first order where they would be expected to appear. This agrees with the results obtained both in [12] and in [9] (see also [13]). In all of those papers, an effective approach was used to provide the argument of vanishing gaugino masses. Here we provide a derivation of this result based on the precise cancellation between the two diagrams contributing to the gaugino mass. This cancellation takes place for any supersymmetry breaking current correlator. Hence, the cancellation is a result which does not depend on the existence of a hidden sector spurion encoding supersymmetry breaking, nor on the specific supersymmetry breaking mechanism occurring in the hidden sector. (Note that this cancellation also invalidates the possibility for unsuppressed gaugino mass in one of the string-inspired quiver gauge theory models discussed in the final section of [11].)

The sfermion masses on the other hand do not vanish at the first order in which they are expected to appear, namely, forth order in both the hidden and the visible gauge coupling $\left(g_{h}^{4} g_{v}^{4}\right)$. We provide an expression for these masses which is very reminiscent of the one appearing in general gauge mediation, though there is a complicated kernel appearing in the momentum integral over the hidden sector correlators. This kernel has two effects. Firstly it reverses the sign of the supertrace (i.e., if the hidden sector would have given tachyonic sfermion masses in a direct gauge mediation scenario, it will give positive squared masses in a semi-direct gauge mediation scenario). Secondly, it has a soft behavior at low momenta, and a mild logarithmic growth at large momenta, such that the sfermion masses are safely under control and finite, even though we generically have a non-vanishing supertrace in the messenger sector. This is to be confronted with [14] where a messenger supertrace was introduced by hand and led to UV divergent (and hence UV sensitive) sfermion masses, due to the fact that once some soft scalar masses are introduced, the others necessarily undergo renormalization.

One might conclude that semi-direct gauge mediation, having almost vanishing 
gaugino masses, is not phenomenologically interesting. On the contrary, we will argue that it can be quite useful when combined with other mechanisms of mediation of supersymmetry breaking, either with a single or multiple hidden sectors. In particular, one could think of combining semi-direct gauge mediation with anomaly mediation (with a sequestered hidden sector) [15]. As we shall see, the sfermion mass contribution from SDGM can stabilize the otherwise tachyonic sleptons arising in the simplest models of anomaly mediation. Interestingly, contrary to what one may superficially imagine, it turns out that in this scenario no substantial fine-tuning is required to accomplish such a welcome conspiracy between the two competing effects. We also discuss another scenario where one combines SDGM with models of direct gauge mediation. As we will show in detail, at fixed hidden sector, SDGM and direct gauge mediation provide opposite signs for the squared sfermion masses. Hence in this case SDGM can be useful both in combination with models with tachyonic sfermions, as before, or in models with suppressed gauginos to make the MSSM sparticle spectrum all of the same order (or even to invert the hierarchy between sfermions and gauginos). In both these cases, however, differently from anomaly mediation, generically one would need a fine tuning. For related work on conformal gauge mediation, see [16].

The rest of this paper is organized as follows. In section 2 we present the semidirect gauge mediation adapted version of the GGM formalism and explain how to use it to compute the visible mass spectrum. In section 3 we compute the gaugino masses and in section 4 the sfermion masses. We end in section 5 discussing the possible phenomenological relevance of semi-direct gauge mediation suggested by our analysis.

\section{General set up for semi-direct gauge mediation}

The models we are considering are characterized by three building blocks:

- A visible sector with gauge group $G_{v}$ (the MSSM or any extension thereof).

- A hidden supersymmetry breaking sector containing, besides confining gauge groups driving dynamical supersymmetry breaking, a global continuous symmetry group $G_{h}$, which is then weakly gauged. 
- A pair of messenger superfields $\Phi$ and $\tilde{\Phi}$ in the bi-fundamentals of $G_{v}$ and $G_{h}$, having a supersymmetric mass $m$ but no other superpotential interactions.

In this section, for simplicity, we assume that both $G_{h}$ and $G_{v}$ are $U(1)$ factors. All our results easily generalize to arbitrary gauge groups by adding the appropriate group theory factors. A pictorial representation of the SDGM scenario is reported in figure 1.

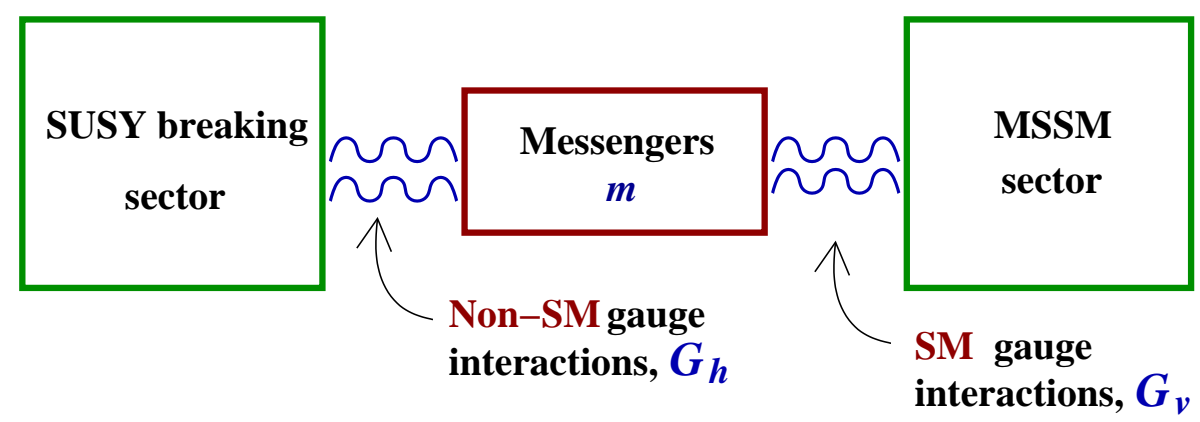

Figure 1: A schematic picture of semi-direct gauge mediation. The gauge group $G_{h}$ is singled-out within the hidden sector as the subgroup of the hidden gauge group to which the messenger superfields couple. Messengers have a supersymmetric mass $m$.

In the limit where the gauge coupling $g_{h}$ of the gauge group $G_{h}$ is sent to zero, the whole system separates into two completely decoupled ones: the supersymmetry breaking sector and a supersymmetry preserving sector comprising the messenger sector and the MSSM fields. This property allows us to use an approach similar to [3], as we can parametrize all of the supersymmetry breaking effects through the correlators of the global $G_{h}$ currents. Namely, we can write, in momentum space 1

$$
\begin{aligned}
\left\langle J^{h}(p) J^{h}(-p)\right\rangle & =C_{0}^{h}\left(p^{2} / M^{2}\right), \\
\left\langle j_{\alpha}^{h}(p) \bar{j}_{\dot{\alpha}}^{h}(-p)\right\rangle & =p_{\mu} \sigma_{\alpha \dot{\alpha}}^{\mu} C_{1 / 2}^{h}\left(p^{2} / M^{2}\right), \\
\left\langle j_{\alpha}^{h}(p) j_{\beta}^{h}(-p)\right\rangle & =\epsilon_{\alpha \beta} M B^{h}\left(p^{2} / M^{2}\right), \\
\left\langle j_{\mu}^{h}(p) j_{\nu}^{h}(-p)\right\rangle & =\left(p_{\mu} p_{\nu}-p^{2} \eta_{\mu \nu}\right) C_{1}^{h}\left(p^{2} / M^{2}\right),
\end{aligned}
$$

where $J^{h}, j_{\alpha}^{h}$ and $j_{\mu}^{h}$ are respectively the scalar, spinor and vector components of the current superfield $\mathcal{J}^{h}$, and $M$ is a typical scale of the dynamical supersymmetry

\footnotetext{
${ }^{1}$ We use a slightly different sign convention with respect to [3], see Appendix A.
} 
breaking sector. If there is more than one scale in that sector, the hierarchies will be encoded in small numerical constants in the functions $C_{s}^{h}$ and $B^{h}$. Additionally, we could also have a non vanishing one-point function for $J^{h}$

$$
\left\langle J^{h}\right\rangle=D^{h}
$$

This expectation value is identified with a non zero $D$-term in the gauge group $G_{h}$, which is then spontaneously broken. Our approach can accommodate easily such a situation, provided the group $G_{h}$ is weakly gauged and the corresponding Higgs generated masses can be treated perturbatively (as in [17]). However we will actually find that such $D^{h}$ will not contribute to the soft masses at leading order.

The complete Lagrangian of the model reads

$$
\begin{aligned}
\mathcal{L}= & \mathcal{L}_{\mathrm{MSSM}}+\int d^{4} \theta\left(\Phi^{\dagger} e^{g_{v} V_{v}+g_{h} V_{h}} \Phi+\tilde{\Phi} e^{-g_{v} V_{v}-g_{h} V_{h}} \tilde{\Phi}^{\dagger}\right) \\
& +\int d^{2} \theta m \Phi \tilde{\Phi}+\int d^{2} \theta \operatorname{tr} \mathcal{W}_{h}^{2}+\text { h.c. }+\int d^{4} \theta g_{h} V_{h} \mathcal{J}^{h}
\end{aligned}
$$

where, with obvious notation, $g_{h}$ is the gauge coupling of the gauge group $G_{h}$, and $g_{v}$ that of the visible sector gauge group $G_{v}$. The last term in (3) represents the first order coupling to the supersymmetry breaking dynamics encoded in (10). There are, of course, additional non linear terms required by gauge invariance and supersymmetry.

In contrast, in general gauge mediation one writes for the same Lagrangian2

$$
\mathcal{L}=\mathcal{L}_{\mathrm{MSSM}}+\int d^{4} \theta g_{v} V_{v} \mathcal{J}^{v}
$$

In turn one encodes all the mediation of supersymmetry breaking to the visible gauge sector in the functions $C_{s}^{v}$ and $B^{v}$ characterizing the correlators of the various components of $\mathcal{J}^{v}$. We thus see that we have in this class of models a sort of tumbling where the functions $C_{s}^{v}$ and $B^{v}$ are eventually determined in terms of the functions $C_{s}^{h}$ and $B^{h}$. This will be our approach in computing the soft masses of the MSSM.

It is rather straightforward to write the general structure of the Feynman diagrams allowing us to extract $C_{s}^{v}$ and $B^{v}$ from $C_{s}^{h}$ and $B^{h}$. What we have to compute are radiative corrections to the two-point functions of the fields in the visible gauge

\footnotetext{
${ }^{2}$ The superscript $v$ in $\mathcal{J}^{v}$ refers to the fact that the currents couple to the visible gauge fields.
} 
sector. The only relevant radiative corrections will be those involving messenger fields, which in turn will be corrected by virtual particles of the gauge group $G_{h}$. In order to have supersymmetry breaking at all, the propagators of the latter must include the insertion of a hidden current correlator.

In figure 2 we have drawn the five topologically distinct Feynman diagrams entering the computation.

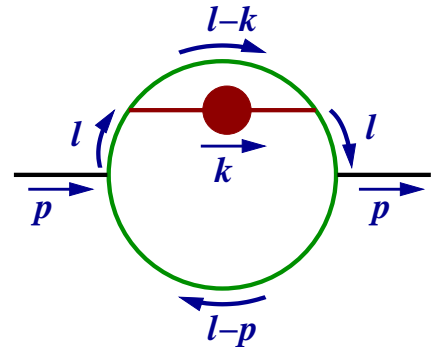

$a$

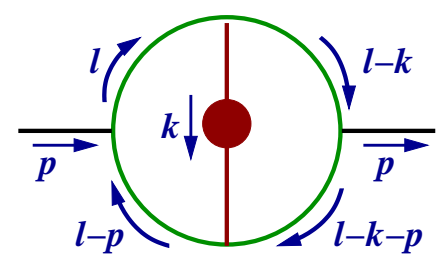

$b$

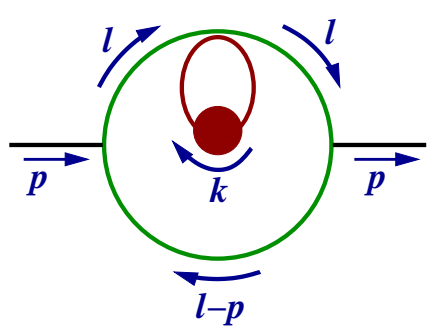

$c$

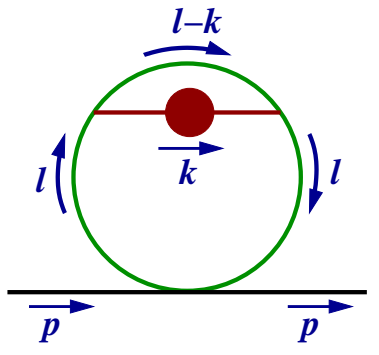

$d$

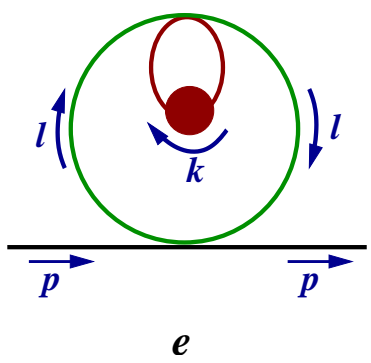

Figure 2: The five topologically inequivalent structures contributing to the visible GGM functions. External lines, which carry momentum $p$, correspond to $G_{v}$-fields (i.e. fields belonging to the visible gauge group). Internal lines carrying momentum $k$ correspond to $G_{h}$-fields (i.e. fields belonging to the hidden sector gauge group $G_{h}$ ) and have attached a blob which encodes the exact hidden sector non-supersymmetric correction to the corresponding propagators. Finally, all other internal lines correspond to messenger fields.

The external lines are those of the $G_{v}$-fields (the MSSM gauge bosons, gauginos and auxiliary $D$-fields). Internal lines carrying momentum $k$ are associated to the fields belonging to the gauge group $G_{h}$ degrees of freedom, and hence will also involve a correlator insertion. All other internal lines are messenger lines, either scalar or 
fermionic. All these diagrams have two explicit loops, plus an additional loop factor coming from the hidden correlator insertion. Hence they all scale like $g_{v}^{2} g_{h}^{4}$.

A first observation is that while the diagrams of topology $a, c, d$ and $e$ can be effectively taken into account as one loop diagrams with corrected messenger lines (i.e. they involve only messenger two-point functions), the diagram with topology $b$ is intrinsically two loops since it involves a messenger four-point function. The contribution of these latter diagrams cannot be encoded in an effective approach such as the one of [14]. As we will see, in computing the visible gaugino masses, there is a dramatic cancellation between the two contributions from the diagrams $a$ and $b$.

A second important observation is that the correlator encoded by the function $B$, which is complex, is on a different footing with respect to the others, which are real. By carefully keeping track of the chiral structure of each diagram, one can see that $B^{v}$ is a function of $B^{h}$ only, while the $C_{s}^{v}$ are functions of a linear combination of the $C_{s}^{h}$.

The knowledge of the functions $C_{s}^{h}$ and $B^{h}$ and, via tumbling, of the functions $C_{s}^{v}$ and $B^{v}$, completely determines the soft masses in the MSSM Lagrangian (3). In what follows, we will compute, using the above formalism, both gaugino and sfermion masses to leading order. Obviously, being a model of gauge mediation, general SDGM obeys, if considered in isolation from other mechanisms, the same sfermion sum rules of GGM [3].

\section{Vanishing of gaugino masses}

In this section we compute the gaugino masses. For this computation, the diagrams of figure 2 are really the end of the story since the external lines are nothing but the gauginos themselves, and we can set the external momentum to be $p=0$.

Of the five topologically inequivalent structures of figure 2 only two contribute to the gaugino masses. Indeed, diagrams $d$ and $e$ appear only if the external lines are vector bosons. Diagram $c$ appears only if the internal line (the one with the blob attached) is a vector bosons, and diagrams of this type are prevented from contributing to gaugino masses by chirality. The only relevant ones are then those 
of type $a$ and $b$. Their precise structure is depicted in figure 3 .

The first one is the same as the one discussed in [10] and evaluates to 3

$$
m_{\lambda}^{a}=-16 g_{v}^{2} g_{h}^{4} \int \frac{d^{4} k}{(2 \pi)^{4}} \int \frac{d^{4} l}{(2 \pi)^{4}} \frac{m^{2} M B^{h}\left(k^{2} / M^{2}\right)}{k^{2}\left(l^{2}+m^{2}\right)^{3}\left[(l-k)^{2}+m^{2}\right]} .
$$

These are Wick rotated expressions and the conventions used for the Euclidean propagators are summarized in Appendix A. We have factors of 4 coming from the four Yukawa vertices, 2 coming from the trace on the internal fermion loop (which also gives the overall - sign), and 2 from the messenger multiplicity.
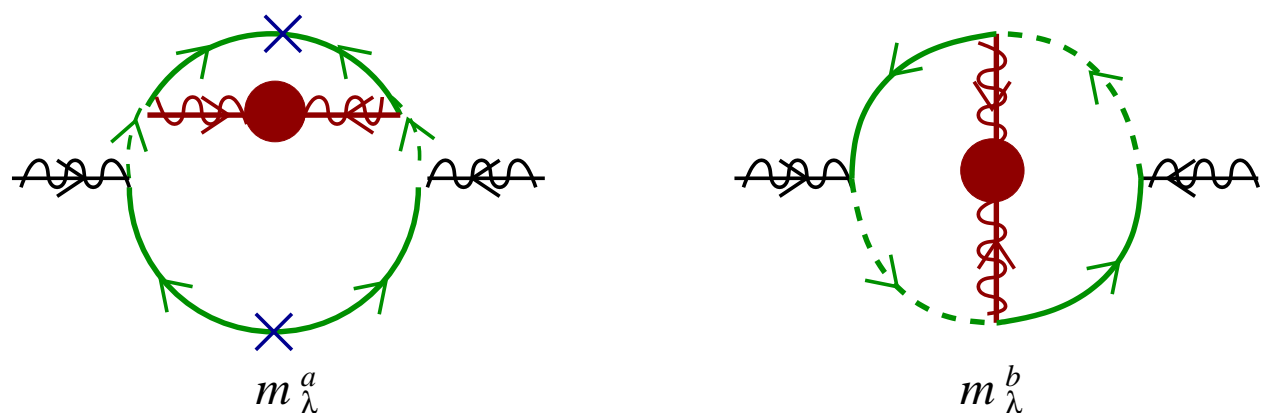

Figure 3: The diagrams contributing to the gaugino mass. The left one has two (supersymmetric) mass insertions, each one represented by a cross on the corresponding messenger fermionic line.

The second diagram, of type $b$, gives the following contribution

$$
m_{\lambda}^{b}=8 g_{v}^{2} g_{h}^{4} \int \frac{d^{4} k}{(2 \pi)^{4}} \int \frac{d^{4} l}{(2 \pi)^{4}} \frac{l \cdot(l-k) M B^{h}\left(k^{2} / M^{2}\right)}{k^{2}\left(l^{2}+m^{2}\right)^{2}\left[(l-k)^{2}+m^{2}\right]^{2}} .
$$

The factors are as before, except for the missing -2 since we have a continuous fermionic line instead of a fermion loop. Obviously, we have that

$$
m_{\lambda}=m_{\lambda}^{a}+m_{\lambda}^{b}
$$

In the two expressions above, the integral over the $l$-momentum can be done analytically by standard techniques. We can then write

$$
m_{\lambda}^{a, b}=8 g_{v}^{2} g_{h}^{4} \frac{M}{m^{2}} \int \frac{d^{4} k}{(2 \pi)^{4}} \frac{1}{k^{2}} L^{a, b}\left(k^{2} / m^{2}\right) B^{h}\left(k^{2} / M^{2}\right),
$$

\footnotetext{
${ }^{3}$ For convenience we drop the gauge theory factor common to both diagrams. Our result is valid for any gauge group.
} 
where the two kernels are given by

$$
\begin{aligned}
L^{a}\left(k^{2} / m^{2}\right) & =-2 m^{4} \int \frac{d^{4} l}{(2 \pi)^{4}} \frac{1}{\left(l^{2}+m^{2}\right)^{3}\left[(l-k)^{2}+m^{2}\right]}, \\
L^{b}\left(k^{2} / m^{2}\right) & =m^{2} \int \frac{d^{4} l}{(2 \pi)^{4}} \frac{l \cdot(l-k)}{\left(l^{2}+m^{2}\right)^{2}\left[(l-k)^{2}+m^{2}\right]^{2}} .
\end{aligned}
$$

Evaluating the two integrals above we find

$$
L^{a}(x)=-L^{b}(x)=-\frac{1}{32 \pi^{2}}\left(\frac{1}{x}+\frac{1}{x+4}-\frac{4 f(x)}{x(x+4)}\right),
$$

where 4

$$
f(x)=\frac{4}{\sqrt{x(x+4)}} \operatorname{arctanh} \sqrt{\frac{x}{x+4}}
$$

and $x=k^{2} / m^{2}$. This result means that the total kernel in the expression for the gaugino mass

$$
m_{\lambda}=8 g_{v}^{2} g_{h}^{4} \frac{M}{m^{2}} \int \frac{d^{4} k}{(2 \pi)^{4}} \frac{1}{k^{2}} L\left(k^{2} / m^{2}\right) B^{h}\left(k^{2} / M^{2}\right)
$$

vanishes, since

$$
L(x)=L^{a}(x)+L^{b}(x)=0 .
$$

Hence, $m_{\lambda}=0$ for any function $B^{h}$, at this order. The fact that the gaugino mass was vanishing at leading order in this class of models was first noted in [12] where an effective argument based on wave function renormalization was given. There it is also shown that the visible gaugino first obtains a mass at order $g_{h}^{4} g_{v}^{6}$. This effect has been dubbed gaugino screening.

Here we have re-derived this important result in a different way. We have shown explicitly how the cancellation arises, which was not obvious from the start (indeed, even a posteriori, the cancellation would seem rather miraculous if we did not have an independent argument in favor of it taking place). Also, and perhaps more importantly, the proof that the kernel $L(x)$ is zero means that the cancellation does not depend on $B^{h}$, and hence applies to any model of supersymmetry breaking

\footnotetext{
${ }^{4}$ For the record, note that the same function $f(x)$ appears when one evaluates the function $B^{v}$ in a minimal gauge mediation scenario $W=\left(m+\theta^{2} F\right) \Phi \tilde{\Phi}$, namely $B^{v}\left(p^{2}\right)=$ $\left(1 / 32 \pi^{2}\right)\left(F / m^{2}\right) f\left(p^{2} / m^{2}\right)$.
} 
that is responsible for the presence of $B^{h}$. This is a counterpart to the argument given in [12] which hinges on the capability of effectively encoding the breaking of supersymmetry in a spurion field, and on the assumption that there are hidden messengers mediating supersymmetry breaking to the gauge group $G_{h}$.

\section{Sfermion masses}

We now turn to the computation of the general expression for the MSSM sfermion masses. In this case the diagrams listed in figure 2 are to be inserted into the self-energy radiative corrections to the scalar propagators. Many such contributions should be computed in this case since now the external lines of the diagrams would not only have gauginos but also gauge bosons and D-fields of the visible gauge group $G_{v}$.

In this section we promote $G_{v}$ to the MSSM gauge group, and consider the case where the messengers form a complete $S U(5)$ multiplet of index $\ell=1$ for each of the MSSM gauge group 5 . We still consider $G_{h}=U(1)$ since more general cases can be easily accommodated by inserting the appropriate group theory factors. In this case the parameter space spanned by the sfermion masses is contained in that of minimal gauge mediation in the sense that all square masses are proportional to one and the same dimension-full parameter. However, the dependence of this parameter on the dynamics of the hidden sector is quite different from the one arising in minimal gauge mediation and this is the issue we are going to analyze.

For ease of notation we define, for each type of sfermion, an effective coupling

$$
g_{v}^{4}=g_{1}^{4} c_{2}[U(1)]+g_{2}^{4} c_{2}[S U(2)]+g_{3}^{4} c_{2}[S U(3)]
$$

in terms of the $S U(3) \times S U(2) \times U(1)$ coupling constants $g_{i}$ of the Standard Model and the Casimir invariants for each of the sfermion representations.

A general expression for the mass square was given in [3]

$$
m_{s f}^{2}=-g_{v}^{4} \int \frac{d^{4} p}{(2 \pi)^{4} p^{2}}\left(C_{0}^{v}\left(p^{2} / M^{2}\right)-4 C_{1 / 2}^{v}\left(p^{2} / M^{2}\right)+3 C_{1}^{v}\left(p^{2} / M^{2}\right)\right) .
$$

\footnotetext{
${ }^{5}$ For $U(1)_{Y}$ the "index" is customarily defined as $\ell=6 Y^{2} / 5$ and the "casimir" $c_{2}=3 Y^{2} / 5$.
} 
Separately, each of the functions $C_{s}^{h}$ and $C_{s^{\prime}}^{v}$ is logarithmically UV-divergent, but all divergences cancel in the final linear combinations. In what follows, we thus only consider the finite parts. Our first goal is to compute the $C_{s^{\prime}}^{v}$ in terms of the $C_{s}^{h}$, and this is exactly what the diagrams in figure 2 do. Obviously there are now two scales $\left(M\right.$ and $m$ ) on which $C_{s^{\prime}}^{v}$ depends. Each of the three functions $C_{s^{\prime}}^{v}$ is expressed as linear combination of contributions from the three functions $C_{s}^{h}$

$$
\begin{aligned}
& C_{s^{\prime}}^{v}\left(p^{2} / M^{2}, m^{2} / M^{2}\right)=\int \frac{d^{4} l}{(2 \pi)^{4}} \frac{d^{4} k}{(2 \pi)^{4}}\left(F_{s^{\prime}, 0}(l, k, p, m) C_{0}^{h}\left(k^{2} / M^{2}\right)-\right. \\
& \left.4 F_{s^{\prime}, 1 / 2}(l, k, p, m) C_{1 / 2}^{h}\left(k^{2} / M^{2}\right)+3 F_{s^{\prime}, 1}(l, k, p, m) C_{1}^{h}\left(k^{2} / M^{2}\right)\right)
\end{aligned}
$$

where we have introduced a series of functions $F_{s^{\prime}, s}(l, k, p, m)$ that can be computed explicitly from the diagrams in Figure 2, The four-momentum $k$ always denotes the momentum going through the current-current correlators in the hidden sector. To obtain the sfermion masses we must then add the three functions $C_{s^{\prime}}^{v}$ according to (16) and integrate over the four-momentum $p$. By switching the integrals, leaving the one over $k$ for last, and combining the various functions $F_{s^{\prime}, s}(l, k, p, m)$ we can easily rewrite the sfermion masses as

$$
\begin{aligned}
m_{s f}^{2}= & \frac{g_{v}^{4} g_{h}^{4}}{(4 \pi)^{4}} \int \frac{d^{4} k}{(2 \pi)^{4} k^{2}}\left(K_{0}\left(k^{2} / m^{2}\right) C_{0}^{h}\left(k^{2} / M^{2}\right)-\right. \\
& \left.4 K_{1 / 2}\left(k^{2} / m^{2}\right) C_{1 / 2}^{h}\left(k^{2} / M^{2}\right)+3 K_{1}\left(k^{2} / m^{2}\right) C_{1}^{h}\left(k^{2} / M^{2}\right)\right)
\end{aligned}
$$

where

$$
\begin{aligned}
& \frac{g_{h}^{4}}{(4 \pi)^{4} k^{2}} K_{s}\left(k^{2} / m^{2}\right)= \\
& -\int \frac{d^{4} l d^{4} p}{(2 \pi)^{8} p^{2}}\left(F_{0, s}(l, k, p, m)-4 F_{1 / 2, s}(l, k, p, m)+3 F_{1, s}(l, k, p, m)\right) .
\end{aligned}
$$

The expressions $K_{s}\left(k^{2} / m^{2}\right)$ represent three (a priori independent) scalar "kernels" depending only on the messenger sector and thus exactly computable irrespectively of what strong dynamics is ultimately responsible for supersymmetry breaking. Recalling that in the supersymmetric limit all $C_{s}^{h}$ are equal and $m_{s f}^{2}=0$, it follows that the weighted sum of the three kernels must vanish: $K_{0}-4 K_{1 / 2}+3 K_{1}=0$. We have computed explicitly the first two of them and found them to be the same 
$\left(K_{0}=K_{1 / 2} \equiv K\right)$, thus implying that the full contribution to the sfermion masses can be written as

$$
m_{s f}^{2}=\frac{g_{v}^{4} g_{h}^{4}}{(4 \pi)^{6}} \int d k^{2} K\left(k^{2} / m^{2}\right)\left(C_{0}^{h}\left(k^{2} / M^{2}\right)-4 C_{1 / 2}^{h}\left(k^{2} / M^{2}\right)+3 C_{1}^{h}\left(k^{2} / M^{2}\right)\right)
$$

The kernel $K(x)$ is given by a sum of integrals over two loop momenta, which are exactly known. We relegate to Appendix B the details of the computation. Suffices here to say that in this case the diagrams of type $b$ involving the messenger four point function are necessary for consistency, for instance to obtain a transverse vectorial current two point function (i.e. a well-defined $C_{1}^{v}$ ).

$K(x)$ is a positive function and has the following asymptotic behavior

$$
\begin{aligned}
K(x) & =\frac{5}{18} x^{2}-\frac{137}{1350} x^{3}+\frac{5437}{176400} x^{4}+\mathcal{O}\left(x^{5}\right) \text { for } x \rightarrow 0 \\
K(x) & \sim \gamma \log x \text { for } x \rightarrow \infty
\end{aligned}
$$

where we have estimated numerically $\gamma \sim 14.4$. A plot of the kernel at large $x$ is given in figure 4 .

From the behavior of the kernel it is clear that the $m_{s f}^{2}$ will always be finite since the weighted sum of the $C_{s}^{h}$ is soft enough at large momenta, as noted in [3]. Note however some important facts.

First of all, in our set up we have a non vanishing supertrace in the messenger sector. According to the argument of [14 this fact, by itself, leads to an enhancement of sfermion squared masses proportional to the logarithm of a UV scale. Here we do not see any such dangerous enhancement. Since the theory is renormalizable and no soft terms appear in the bare Lagrangian, there cannot be counterterms for the sfermion masses and the only two scales of the problem are $M$, the hidden sector supersymmetry breaking scale, and $m$, the messenger supersymmetric mass.

Secondly, due to the fact that $K(x)$ is positive, the sfermion squared masses will be of the opposite sign with respect to the case where the same hidden supersymmetric sector is directly coupled to the MSSM, cfr. eqs. (16) and (20). As we will see in the next section, this is something useful when trying to use SDGM in concrete phenomenological models. 


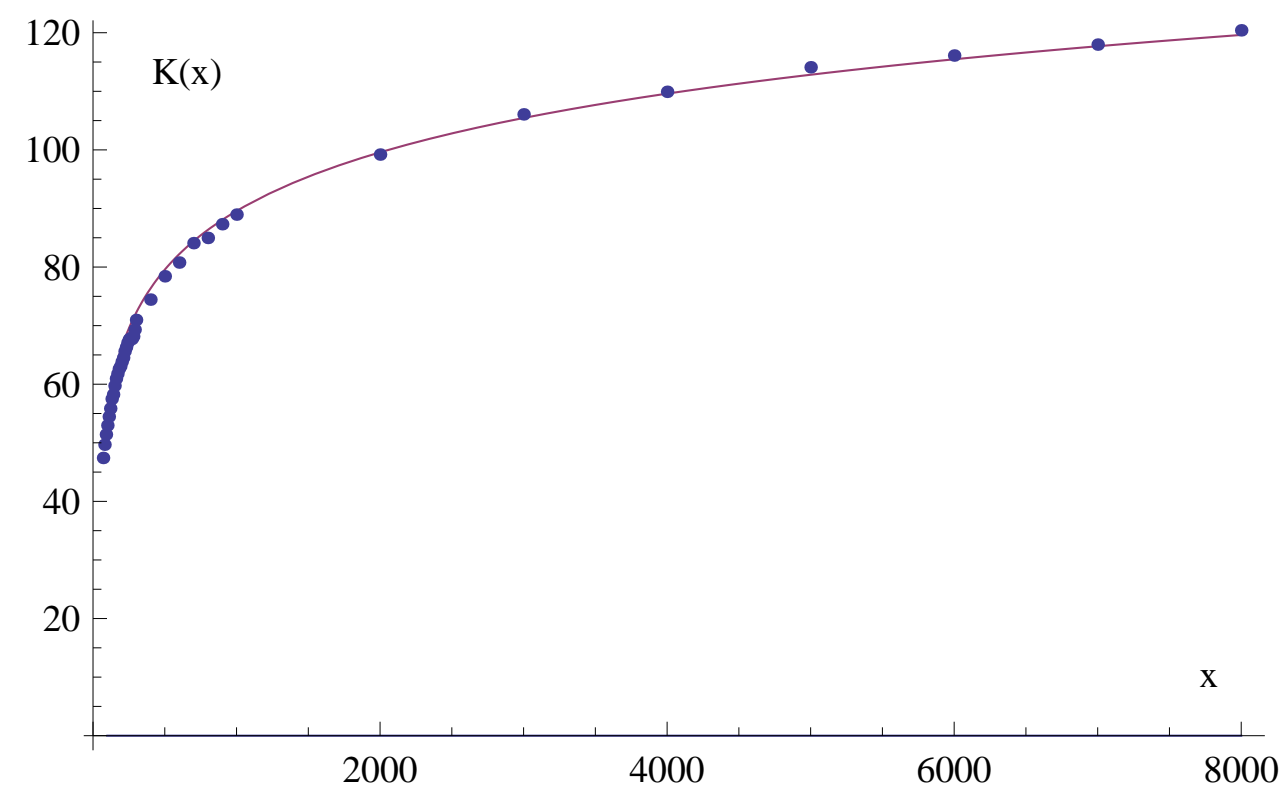

Figure 4: Numerical estimate of the kernel $K(x)$ for large $x$. The fit is represented by the curve $14.4 \log x-10.3$. The numerical errors are about $5 \%$ - quite sufficient for the simple estimates done in this paper.

As a last remark, we note that the fact the kernel vanishes for small momenta leads to a vanishing contribution to the sfermion masses from $D$-terms, if such terms are present. One would expect a first contribution from them at order $\left(D^{h}\right)^{2}$, since the contribution at linear order vanishes after summing over both charge conjugate messengers. Two $D$-tadpole insertions can actually be encoded in $C_{0 D}^{h}\left(k^{2}\right) \sim\left(D^{h}\right)^{2} \delta^{4}(k)$ (indeed, two $D$-tadpoles are equivalent to a $D$-line cut in two, and hence with no momentum flowing through it). Obviously, this contribution vanishes when multiplied by $K(x) \sim x^{2}$. This fact can be extracted from the general expression given in [14] by tuning the parameters so that only $D$-terms contribute to the messenger mass matrix. This precise situation and its phenomenology was analyzed more recently for instance in [18] where next-to-leading order corrections were discussed.

We can give a rough estimate of the scaling of $m_{s f}^{2}$ as a function of $M$ and $m$, in the two opposite hierarchical limits. We will assume that the weighted sum is a simple step function (which can mimic, roughly, the soft behavior previously 
discussed)

$$
\left(C_{0}^{h}-4 C_{1 / 2}^{h}+3 C_{1}^{h}\right)\left(k^{2} / M^{2}\right) \sim \frac{1}{16 \pi^{2}} \Theta\left(1-k^{2} / M^{2}\right),
$$

with a proportionality factor of either sign depending on the specific hidden sector 6 .

In the limit of heavy messengers we get

$$
m_{s f}^{2} \sim \frac{\alpha_{v}^{2} \alpha_{h}^{2}}{(4 \pi)^{4}} \frac{5}{54} \frac{M^{6}}{m^{4}} \quad m \gg M,
$$

while in the opposite limit of light messengers

$$
m_{s f}^{2} \sim \frac{\alpha_{v}^{2} \alpha_{h}^{2}}{(4 \pi)^{4}} \gamma M^{2} \log \left(\frac{M^{2}}{m^{2}}\right) \quad m \ll M,
$$

where we have defined $\alpha_{h, v}=g_{h, v}^{2} / 4 \pi$. While we see a signal of the log-enhancement of [14] in the limit $m \ll M$, we do not see anything like it in the opposite limit.

For completeness, we can estimate the contributions to the mass matrix of the messengers in the limit $m \ll M$ where the radiative corrections can be comparable to the supersymmetric mass. In this limit we can treat $m$ as a small perturbation and write the following expressions

$$
\begin{aligned}
& m_{d}^{2}=-g_{h}^{4} \int \frac{d^{4} k}{(2 \pi)^{4} k^{2}}\left(C_{0}^{h}\left(k^{2} / M^{2}\right)-4 C_{1 / 2}^{h}\left(k^{2} / M^{2}\right)+3 C_{1}^{h}\left(k^{2} / M^{2}\right)\right), \\
& m_{o}^{2}=g_{h}^{4} \int \frac{d^{4} k}{(2 \pi)^{4} k^{2}} \frac{m M B^{h}\left(k^{2} / M^{2}\right)}{k^{2}}
\end{aligned}
$$

where $m_{d}^{2}$ and $m_{o}^{2}$ are respectively the contributions to the diagonal and off-diagonal elements of the messenger mass matrix. The supertrace over the messenger sector is proportional to $m_{d}^{2}$.

Using the estimate (23) and a similar one for $B^{h}$, we obtain the estimates for $m \ll M$

$$
m_{d}^{2} \sim \frac{\alpha_{h}^{2}}{(4 \pi)^{2}} M^{2}, \quad m_{o}^{2} \sim \frac{\alpha_{h}^{2}}{(4 \pi)^{2}} m M \log \left(\frac{M^{2}}{m^{2}}\right) .
$$

The sign of $m_{d}^{2}$ (and thus of the supertrace) is the opposite of the sign in the sfermion masses (25).

\footnotetext{
${ }^{6}$ Again, for the record, we note that in a minimal gauge mediation scenario we would obtain $\left(C_{0}-4 C_{1 / 2}+3 C_{1}\right)\left(p^{2}\right)=\left(1 / 16 \pi^{2}\right)\left(2|F|^{2} / m^{4}\right) f^{\prime}\left(p^{2} / m^{2}\right)$, where $f^{\prime}(x)$ is the derivative of the function $f(x)$ that we encountered previously, and is everywhere negative.
} 
We conclude that even if the scale of the supersymmetry breaking sector is much higher than the supersymmetric mass of the messengers, it is generally possible to avoid tachyonic eigenvalues in their mass matrix due to the suppression in $\alpha_{h}$ of the radiative corrections to the messenger mass.

\section{Phenomenological uses of semi-direct gauge me- diation}

We now comment on the possible phenomenological relevance of the class of models considered in this paper. We have seen that the sparticle spectrum produced is essentially one of a strong hierarchy between the sfermions, which are heavy, and the gauginos which are very light (massless at the order considered above). This is clearly not a satisfying spectrum in itself. Additionally, since there is an extra loop factor $\alpha_{h}$ in the expression for the sfermion masses, the gravitino is going to be a factor of $1 / \alpha_{h}$ heavier than in an ordinary gauge mediation model yielding the same values of $m_{s f}$.

However, since SDGM gives a contribution mainly to sfermion masses, it can be useful if combined with other supersymmetry breaking mechanisms which provide gaugino masses but tachyonic sfermions. We review below two such situations, in which the other mechanisms are respectively anomaly mediation (AM) and direct gauge mediation (DGM).

We first analyze the scenario where SDGM could address the negative squared sfermion mass problem of anomaly mediation [15]. We consider the simple set up where AM and SDGM have the same supersymmetry breaking sector, and hence the same supersymmetry breaking scale $M$. Anomaly mediation gives a gaugino mass of the order

$$
m_{\lambda} \sim \frac{\alpha_{v}}{4 \pi} \frac{M^{2}}{M_{p l}} .
$$

Assuming a sequestered hidden sector, the slepton masses for the first two genera- 
tions are of the order 7

$$
m_{A M}^{2} \sim-\frac{\alpha_{v}^{2}}{(4 \pi)^{2}} \frac{M^{4}}{M_{p l}^{2}} .
$$

The slepton masses in (30) are tachyonic because of the sign of the beta function coefficient (the contribution from the Yukawa couplings can be ignored for the first two generations).

We can cure the problem of tachyonic sleptons by combining AM with a SDGM model which yields positive sfermion squared masses. Let us denote by $\delta$ the ratio between the SDGM contribution (24) or (25) to the slepton masses and (30). Depending on the messenger scale, we obtain

$$
\delta \equiv\left|\frac{m_{S D}^{2}}{m_{A M}^{2}}\right| \sim\left\{\begin{array}{cl}
\frac{\alpha_{h}^{2}}{(4 \pi)^{2}} \gamma \frac{M_{p l}^{2}}{M^{2}} \log \frac{M^{2}}{m^{2}} & \text { for } m \ll M \\
\frac{\alpha_{h}^{2}}{(4 \pi)^{2}} \frac{5}{54} \frac{M^{2} M_{p l}^{2}}{m^{4}} & \text { for } m \gg M
\end{array}\right.
$$

We require $1<\delta<10$ in order for the SDGM to give a contribution to sfermion masses which is larger than AM but of the same order. This can be achieved in both regimes while staying at weak coupling in $\alpha_{h}$.

First of all, in order to have gaugino masses (29) at the $\mathrm{TeV}$ scale, we need to take $M \sim 10^{12} \mathrm{GeV}$.

In the first case $(m \ll M)$, assuming $\frac{\alpha_{h}}{4 \pi} \sim 10^{-7}$ gives a sensible soft spectrum where the messenger mass can be anywhere in the range $10^{5}-10^{10} \mathrm{GeV}$. Note that such a small $G_{h}$ coupling constant could be actually related to the mechanism of sequestering itself. In the second case $(m \gg M)$, one could have for instance $m \sim 10^{14} \mathrm{GeV}$ with $\frac{\alpha_{h}}{4 \pi} \sim 10^{-2}$.

We conclude that SDGM can be successfully combined with AM, in both regimes and with no substantial fine-tuning, and cure the slepton problem (some earlier interesting proposals to cure this problem can be found in [20], and more recently in [21]). It is worth mentioning at this point that such a conspiracy could arise naturally

\footnotetext{
${ }^{7}$ Since we are only interested in order of magnitude estimates, we use a notation similar to (15) and lump all dependence on the visible couplings into $\frac{\alpha_{v}}{4 \pi} \sim 3 \cdot 10^{-3}$. One could be more precise, if needed. For instance, focusing on $U(1)_{Y}$, one has, for the Bino and the right selectron: $m_{\tilde{B}}=(33 / 5) \frac{\alpha_{1}}{4 \pi} \frac{M^{2}}{M_{p l}}$ and $m_{\tilde{e}_{R}}^{2}=-(198 / 25) \frac{\alpha_{1}^{2}}{(4 \pi)^{2}} \frac{M^{4}}{M_{p l}^{2}}$ respectively (see e.g. [19]).
} 
in string theory since SDGM is generic in D-branes embeddings of gauge mediation [11], while anomaly mediation must always be included once gravity effects are considered on D-branes.

We now turn to the study of the combination of SDGM with DGM. We consider again the simple set up where the two mechanisms share the same supersymmetry breaking sector. Note that the currents coupling to the visible gauge group $G_{v}$ and the ones coupling to $G_{h}$ must be different since they couple to different gauge groups. However we work in the approximation where their correlation functions are essentially the same. This is not unnatural if the supersymmetry breaking sector has only one scale or if the two groups arise from the breaking of a larger group. The important point here is that the contribution to the sfermion masses of SDGM has an opposite sign with respect to the DGM one. This comes, as already noticed in section 4, by comparing eqs. (16) and (20), given the positivity of the kernel $K(x)$.

Models of strongly coupled DGM can lead to unsuppressed gaugino masses but negative squared scalar masses. Using the approximation (23) the DGM contribution to the sfermion masses is

$$
m_{D}^{2} \sim-\frac{\alpha_{v}^{2}}{(4 \pi)^{2}} M^{2} .
$$

In this case the positive SDGM contribution could render the sfermion masses non tachyonic, in the limit $m \ll M$ where it can be larger than (32). To understand if the competition between SDGM and DGM can be realized naturally, we need to estimate the ratio of the sfermion mass contributions (25) and (32)

$$
\delta=\left|\frac{m_{S D}^{2}}{m_{D}^{2}}\right| \sim \gamma \frac{\alpha_{h}^{2}}{(4 \pi)^{2}} \log \frac{M^{2}}{m^{2}} .
$$

We would then demand $1<\delta<10$, as before.

Note that, besides the requirement on $\delta$, we have to check that the messengers in the SDGM sector do not become tachyonic due to radiative corrections. In section 4 we estimated the diagonal and off-diagonal corrections to the messengers mass matrix (28) in the limit $m \ll M$. Note that in the case at hand the diagonal radiative correction $m_{d}^{2}$ is negative since the SDGM contribution to sfermion masses is positive.

It is possible, in principle, to satisfy these competing constraints. However, some amount of tuning will be needed in this case. On the one hand, the scale $M$ should 
be larger than $m$ to avoid a too strong $G_{h}$ coupling while keeping $\delta>1$. On the other hand, $M$ should not be too large, in order to avoid tachyons in the messenger sector. The possibility of satisfying both these constraints is not generic and it can only be answered on a case by case basis. However, the possibility of a window where such a mechanism can work is not ruled out 8

An alternative scenario concerns models of DGM which present an MSSM sparticle spectrum where the gaugino mass is suppressed or of the same order of the (positive) sfermion masses. Here the SDGM can provide a negative contribution to the sfermion masses in order to invert this hierarchy. This scenario can be realized similarly as above, however with a fine tuning of $\delta$, i.e. of the coupling constant $\alpha_{h}$. The fact that a fine tuning is needed in order to obtain gauginos more massive than sfermions seems a common feature of gauge mediated models (see for instance [4]).

In conclusion, our preliminary analysis indicates that models of AM+SDGM can naturally lead to a sensible MSSM soft mass spectrum and thus seem promising for phenomenological applications. On the other hand, the SDGM+DGM scenarios that we discussed above can possibly lead to sensible phenomenology only in a small region of the parameter space, if at all.

\section{Acknowledgments}

We thank Ken Intriligator for discussions at the beginning of this project and Andrea Romanino for useful comments on a preliminary version of the draft. The research of R.A. is supported in part by IISN-Belgium (conventions 4.4511.06, 4.4505.86 and 4.4514.08). R.A. is a Research Associate of the Fonds de la Recherche ScientifiqueF.N.R.S. (Belgium). The research of G.F. is supported in part by the Swedish Research Council (Vetenskapsrådet) contract 621-2006-3337. Contribution from the Längmanska Kulturfonden and the Wilhelm och Martina Lundgrens Veteskapsfond are also gratefully acknowledged. A.M. is a Postdoctoral Researcher of FWOVlaanderen. A.M. is also supported in part by FWO-Vlaanderen through project G.0428.06. R.A. and A.M. are supported in part by the Belgian Federal Science

\footnotetext{
${ }^{8}$ Possibly, combining SDGM and DGM with different supersymmetry breaking sectors can ameliorate these problems, at the price of introducing new scales in the model.
} 
Policy Office through the Interuniversity Attraction Pole IAP VI/11.

\section{A Conventions}

We use the following propagators for the messengers

$$
\begin{aligned}
\left\langle\phi(p) \phi^{*}(-p)\right\rangle & =\frac{1}{p^{2}+m^{2}} \\
\left\langle\psi_{\alpha}(p) \bar{\psi}_{\dot{\alpha}}(-p)\right\rangle & =\frac{p_{\mu} \sigma_{\alpha \dot{\alpha}}^{\mu}}{p^{2}+m^{2}} \\
\left\langle\psi_{\alpha}(p) \psi^{\beta}(-p)\right\rangle & =\frac{m \delta_{\alpha}^{\beta}}{p^{2}+m^{2}},
\end{aligned}
$$

while for the hidden gauge sector we have the following propagators, at first order in the insertions of the (supersymmetry breaking) currents

$$
\begin{aligned}
\left\langle D^{h}(p) D^{h}(-p)\right\rangle & =C_{0}^{h}\left(p^{2} / M^{2}\right) \\
\left\langle\lambda_{\alpha}^{h}(p) \bar{\lambda}_{\dot{\alpha}}^{h}(-p)\right\rangle & =-\frac{p_{\mu} \sigma_{\alpha \dot{\alpha}}^{\mu}}{p^{2}} C_{1 / 2}^{h}\left(p^{2} / M^{2}\right) \\
\left\langle\lambda_{\alpha}^{h}(p) \lambda^{h \beta}(-p)\right\rangle & =\frac{M \delta_{\alpha}^{\beta}}{p^{2}} B^{h}\left(p^{2} / M^{2}\right)^{\star} \\
\left\langle A_{\mu}^{h}(p) A_{\nu}^{h}(-p)\right\rangle & =\frac{p_{\mu} p_{\nu}-p^{2} \eta_{\mu \nu}}{p^{4}} C_{1}^{h}\left(p^{2} / M^{2}\right) .
\end{aligned}
$$

In these conventions that use exclusively Weyl spinors, each $\phi^{*} \psi \lambda$ Yukawa vertex comes with a $g \sqrt{2}$ coupling. Note also that

$$
\sigma_{\alpha \dot{\alpha}}^{\mu} \bar{\sigma}^{\nu \dot{\alpha} \beta}+\sigma_{\alpha \dot{\alpha}}^{\nu} \bar{\sigma}^{\mu \dot{\alpha} \beta}=-2 \eta^{\mu \nu} \delta_{\alpha}^{\beta},
$$

and similarly for $\bar{\sigma} \sigma$. We use the $(-+++)$ signature, hence all the above relations are unchanged after Wick rotation.

\section{B Computation of the kernel}

In this appendix we collect some more details concerning the computation of the kernels $K_{s}(x)$, proving, eventually, that $K_{0}(x)=K_{1 / 2}(x)=K_{1}(x)$. Recall that upon use of the tumbling equations (17), the sfermion masses (16) depend on the 
kernels $K_{s}$ as given in (18). To compute the kernels $K_{s}$ one should write down all the graphs contributing to the visible $C_{s^{\prime}}^{v}$ as in the tumbling equations (17). Then one can extract the functions $F_{s^{\prime}, s}$ and integrate them in order to obtain the kernels (19).

Let us focus on the contributions to $K_{0}$, first. Since this is defined as the function multiplying $C_{0}^{h}$ we need to consider all the diagrams giving the dependence of $C_{s}^{v}$ on $C_{0}^{h}$, namely those diagrams depicted in figure 5 .

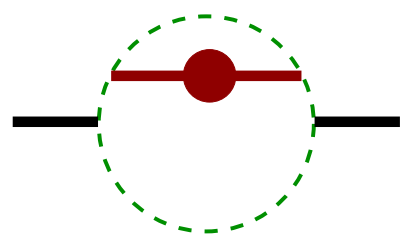

$\mathrm{DD}_{a}$

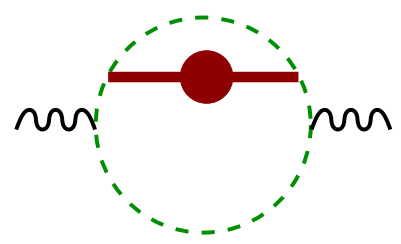

$\mathrm{AD}_{a}$

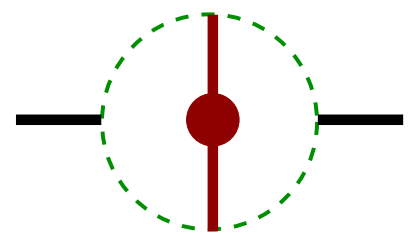

$\mathrm{DD}_{b}$

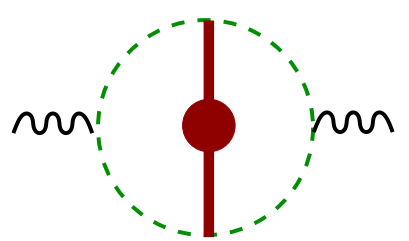

$\mathrm{AD}_{b}$

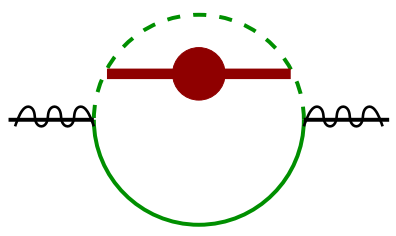

$\lambda \mathrm{D}_{a}$

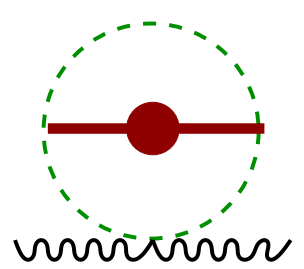

$\mathrm{AD}_{d}$

Figure 5: The diagrams contributing to $K_{0}$. The external lines represent the auxiliary $D$-field, the gaugino and the gauge boson of the visible sector gauge group $G_{v}$. The scalar and spinor messengers (dashed and continuum thin lines, respectively) circulate in the outer loop and the bubble represents the insertion of $C_{0}^{h}$. Each diagram must be counted with the appropriate coefficient representing the different ways messengers can be inserted.

The incoming lines represent the visible gauge particles ( $D$-field, gaugino and gauge boson), the particles going around the external loop are the bosonic and fermionic messengers and, finally, the internal line represents the insertion of the hidden two-point function $\left\langle J^{h}(k) J^{h}(-k)\right\rangle$ on a hidden $D$-line.

To completely specify a diagram one would also need to specify the orientation of the internal lines (including the chirality type for the fermions) and the type 
of messengers $(\Phi$ or $\tilde{\Phi})$. We choose not to write this explicitly in order to keep the notation simple. Each of the six diagrams in figure 5 thus represents a set of diagrams and this is reflected into the numerical coefficients for each contribution. Other numerical coefficients arise from the normalization of the interaction vertices and from the Dirac algebra.

The total contribution for each class of diagrams is given by

$$
\begin{aligned}
D D_{a} & =4 \int \frac{d k^{4} d l^{4}}{(2 \pi)^{8}} \frac{C_{0}^{h}\left(k^{2} / M^{2}\right)}{\left(l^{2}+m^{2}\right)^{2}\left[(l-k)^{2}+m^{2}\right]\left[(l-p)^{2}+m^{2}\right]} \\
D D_{b} & =2 \int \frac{d k^{4} d l^{4}}{(2 \pi)^{8}} \frac{C_{0}^{h}\left(k^{2} / M^{2}\right)}{\left(l^{2}+m^{2}\right)\left[(l-k)^{2}+m^{2}\right]\left[(l-k-p)^{2}+m^{2}\right]\left[(l-p)^{2}+m^{2}\right]} \\
\lambda D_{a} & =-4 \int \frac{d k^{4} d l^{4}}{(2 \pi)^{8}} \frac{(l-p)_{\mu} \sigma_{\alpha \dot{\alpha}}^{\mu} C_{0}^{h}\left(k^{2} / M^{2}\right)}{\left(l^{2}+m^{2}\right)^{2}\left[(l-k)^{2}+m^{2}\right]\left[(l-p)^{2}+m^{2}\right]} \\
A D_{a} & =4 \int \frac{d k^{4} d l^{4}}{(2 \pi)^{8}} \frac{(2 l-p)_{\mu}(2 l-p)_{\nu} C_{0}^{h}\left(k^{2} / M^{2}\right)}{\left(l^{2}+m^{2}\right)^{2}\left[(l-k)^{2}+m^{2}\right]\left[(l-p)^{2}+m^{2}\right]} \\
A D_{b} & =2 \int \frac{d k^{4} d l^{4}}{(2 \pi)^{8}} \frac{(2 l-p)_{\mu}(2 l-2 k-p)_{\nu} C_{0}^{h}\left(k^{2} / M^{2}\right)}{\left(l^{2}+m^{2}\right)\left[(l-k)^{2}+m^{2}\right]\left[(l-k-p)^{2}+m^{2}\right]\left[(l-p)^{2}+m^{2}\right]} \\
A D_{d} & =-4 \int \frac{d k^{4} d l^{4}}{(2 \pi)^{8}} \frac{\eta_{\mu \nu} C_{0}^{h}\left(k^{2} / M^{2}\right)}{\left(l^{2}+m^{2}\right)^{2}\left[(l-k)^{2}+m^{2}\right]} \cdot
\end{aligned}
$$

The notation of the above integrals should be self-evident, for instance $A D$ denotes the contribution of the hidden $D$-field to the visible gauge boson $A$. The subscript refers to the five topologies introduced in figure 2 (only diagrams of topology $a, b$ and $d$ enter the computation of $K_{0}$ ). There are several consistency checks for the above expressions. For instance, one can check that the sum $A D_{a}+A D_{b}+A D_{d}$ obeys the Ward identities.

To obtain $K_{0}$ we need to extract the expression for the contribution to $C_{s}^{v}$ from the diagrams above by comparing (42) with the definition (11). One then inserts the contribution thus obtained into (16). The resulting two-loop integral (in $p$ and $l$ ) defines the kernel $K_{0}\left(k^{2} / m^{2}\right)$. It turns out to be convenient to write everything with a common denominator and express the integral over $l$ in terms of three Feynman parameters. The resulting integrand can be expanded in power series of $k$ and the coefficients (depending on the three Feynman parameters, $p$ and the angle between $p$ and $k$ ) can be fully integrated yielding (21). Alternatively, one can perform a 
numerical integration and obtain the behavior (22) of the kernel for large $k$.

We performed an analogous computation for $K_{1 / 2}$. (We spare the reader the details.) Despite the rather different structure of the contributions, we find that $K_{1 / 2}$ is the same as $K_{0}$. As discussed in section 4, supersymmetry implies that the last kernel $K_{1}$ also be the same as the previous two thus completing the computation and proving that we have one and only one common kernel $K$.

\section{References}

[1] M. Dine and W. Fischler, Phys. Lett. B 110 (1982) 227.

C. R. Nappi and B. A. Ovrut, Phys. Lett. B 113, 175 (1982).

M. Dine and W. Fischler, Nucl. Phys. B 204 (1982) 346.

L. Alvarez-Gaume, M. Claudson and M. B. Wise, Nucl. Phys. B 207, 96 (1982).

[2] G. F. Giudice and R. Rattazzi, Phys. Rept. 322 (1999) 419 arXiv:hep-ph/9801271.

[3] P. Meade, N. Seiberg and D. Shih, arXiv:0801.3278 [hep-ph].

[4] M. Buican, P. Meade, N. Seiberg and D. Shih, JHEP 0903 (2009) 016 arXiv:0812.3668 [hep-ph]].

D. Marques, JHEP 0903 (2009) 038 [arXiv:0901.1326 [hep-ph]].

[5] S. A. Abel, J. Jaeckel and V. V. Khoze, arXiv:0907.0658 [hep-ph].

S. Abel, M. J. Dolan, J. Jaeckel and V. V. Khoze, arXiv:0910.2674 [hep-ph].

[6] M. Dine and A. E. Nelson, Phys. Rev. D 48, 1277 (1993) arXiv:hep-ph/9303230.

M. Dine, A. E. Nelson and Y. Shirman, Phys. Rev. D 51, 1362 (1995) arXiv:hep-ph/9408384].

M. Dine, A. E. Nelson, Y. Nir and Y. Shirman, Phys. Rev. D 53, 2658 (1996) arXiv:hep-ph/9507378. 
[7] E. Poppitz and S. P. Trivedi, Phys. Rev. D 55, 5508 (1997) arXiv:hep-ph/9609529].

N. Arkani-Hamed, J. March-Russell and H. Murayama, Nucl. Phys. B 509, 3 (1998) arXiv:hep-ph/9701286.

H. Murayama, Phys. Rev. Lett. 79, 18 (1997) arXiv:hep-ph/9705271.

S. Dimopoulos, G. R. Dvali, R. Rattazzi and G. F. Giudice, Nucl. Phys. B 510 (1998) 12 arXiv:hep-ph/9705307].

[8] R. Kitano, H. Ooguri and Y. Ookouchi, Rev. D 75 (2007) 045022 |arXiv:hep-ph/0612139|.

C. Csaki, Y. Shirman and J. Terning, JHEP 0705 (2007) 099 arXiv:hep-ph/0612241].

A. Amariti, L. Girardello and A. Mariotti, Fortsch. Phys. 55 (2007) 627 arXiv:hep-th/0701121.

N. Haba and N. Maru, Phys. Rev. D 76 (2007) 115019 arXiv:0709.2945 [hep$\mathrm{ph}]$.

[9] N. Seiberg, T. Volansky and B. Wecht, arXiv:0809.4437 [hep-ph]].

H. Elvang and B. Wecht, JHEP 0906 (2009) 026 [arXiv:0904.4431 [hep-ph]].

[10] L. Randall, Nucl. Phys. B 495 (1997) 37 arXiv:hep-ph/9612426.

C. Csaki, L. Randall and W. Skiba, Phys. Rev. D 57 (1998) 383 arXiv:hep-ph/9707386.

[11] R. Argurio, M. Bertolini, G. Ferretti and A. Mariotti, Phys. Rev. D 80 (2009) 045001 arXiv:0906.0727 [hep-th]].

[12] N. Arkani-Hamed, G. F. Giudice, M. A. Luty and R. Rattazzi, Phys. Rev. D 58 (1998) 115005 arXiv:hep-ph/9803290.

[13] M. Ibe, K. I. Izawa and Y. Nakai, arXiv:0812.4089 [hep-ph].

Z. Komargodski and D. Shih, JHEP 0904, 093 (2009) arXiv:0902.0030 [hepth]]. 
M. Ibe, K. I. Izawa and Y. Nakai, Phys. Rev. D 80 (2009) 035002 arXiv:0907.2970 [hep-ph]].

A. Giveon, A. Katz and Z. Komargodski, JHEP 0907, 099 (2009) arXiv:0905.3387 [hep-th]].

[14] E. Poppitz and S. P. Trivedi, Phys. Lett. B 401 (1997) 38 arXiv:hep-ph/9703246.

[15] L. Randall and R. Sundrum, Nucl. Phys. $\quad$ B $557 \quad$ (1999) 79 arXiv:hep-th/9810155.

G. F. Giudice, M. A. Luty, H. Murayama and R. Rattazzi, JHEP 9812 (1998) 027 arXiv:hep-ph/9810442.

[16] M. Ibe, Y. Nakayama and T. T. Yanagida, Phys. Lett. B 649 (2007) 292 arXiv:hep-ph/0703110.

M. Ibe, Y. Nakayama and T. T. Yanagida, Phys. Lett. B 671 (2009) 378 arXiv:0804.0636 [hep-ph]].

[17] M. Buican and Z. Komargodski, arXiv:0909.4824 [hep-ph].

[18] Y. Nakayama, M. Taki, T. Watari and T. T. Yanagida, Phys. Lett. B 655 (2007) 58 [arXiv:0705.0865 [hep-ph]].

[19] S. P. Martin, arXiv:hep-ph/9709356.

[20] A. Pomarol and R. Rattazzi, JHEP 9905 (1999) 013 arXiv:hep-ph/9903448.

Z. Chacko, M. A. Luty, I. Maksymyk and E. Ponton, JHEP 0004 (2000) 001 arXiv:hep-ph/9905390.

D. E. Kaplan and G. D. Kribs, JHEP 0009 (2000) 048 arXiv:hep-ph/0009195].

[21] J. de Blas, P. Langacker, G. Paz and L. T. Wang, arXiv:0911.1996 [hep-ph]. 